\title{
Editorial: Vibrio Virulence Regulation and Host Interactions
}

\author{
Lixing Huang ${ }^{1 *}$, Yang $\mathrm{Fu}^{2}$, Wenxiang Sun ${ }^{3}$ and Joanna Brzostek ${ }^{4}$ \\ ${ }_{1}^{1}$ Fisheries College, Fujian Engineering Research Center of Aquatic Breeding and Healthy Aquaculture, Jimei University, \\ Xiamen, China, ${ }^{2}$ School of Medicine, Southern University of Science and Technology, Shenzhen, China, ${ }^{3}$ Huntsman Cancer \\ Institute and Department of Pathology, School of Medicine, University of Utah, Salt Lake City, UT, United States, \\ ${ }^{4}$ Department of Microbiology and Immunology, National University of Singapore, Singapore, Singapore
}

Keywords: vibrio, virulence, pathogen-host interactions, Research Topic, bacteria

Editorial on the Research Topic

Vibrio Virulence Regulation and Host Interactions

Vibrio is an ubiquitous bacterium that is widely present in various aquatic and marine habitats; among the more than 100 species of Vibrio described, about 12 can cause human infections, while other Vibrio lead to marine animal infections. Vibrio cholerae can cause cholera, a serious diarrheal disease that is life-threatening if left untreated, and usually spread through contaminated water and personal contact. Non-cholera Vibrio species cause vibriosis - an infection usually are acquired through contacting with seawater or eating raw or undercooked contaminated seafood. Noncholera Vibrio can cause a variety of clinical manifestations, including self-limiting gastroenteritis, wound infection, sepsis, and death. The incidence of vibriosis is on the rise, partly due to the spread of Vibrio spp. favored by climate change and rising sea temperature.

Considering that Vibrio is the leading opportunistic pathogen causing serious infections in humans and marine animals, there is an urgent need to better understand the pathogenic mechanism of Vibrio infections to guide effective prevention and treatments. Meanwhile, preventing and avoiding Vibrio infections and the resulting deaths require a deeper understanding of the mechanisms by which human and animal immune systems recognize and respond to Vibrio. To this end and through this Research Topic of 12 articles including original research and comprehensive reviews, we coalesce the latest advances in the current understanding of Vibrio virulence regulation and host interactions.

lixinghuang@jmu.edu.cn

Lixing Huang

The Research Institute at Nationwide Children's Hospital, United States

\section{${ }^{*}$ Correspondence:}

Specialty section:

This article was submitted to Molecular Bacterial Pathogenesis, a section of the journal

Frontiers in Cellular and

Infection Microbiology

Received: 12 October 2021 Accepted: 18 October 2021 Published: 29 October 2021

Citation:

Huang L, Fu Y, Sun W and Brzostek J (2021) Editorial: Vibrio Virulence Regulation and Host Interactions. Front. Cell. Infect. Microbiol. 11:793464. doi: 10.3389/fcimb.2021.793464

\section{Vibrio cholerae VIRULENCE REGULATION AND HOST INTERACTIONS}

$V$. cholerae is the pathogen causing cholera. It can proliferate in the water environment and infect humans through contaminated food and water. In $V$. cholerae, virulence is a multilocus phenomenon with a large functionally associated network. Ramamurthy et al. extensively summarize the regulation of important virulence factors in $V$. cholerae and the host response in the context of pathogenesis, covering topics such as major toxins produced by $V$. cholerae and their regulation, the control of virulence by the ToxR regulon, the regulation of secretory systems, the host's response to toxins and somatic antigens, as well as the host's inflammatory response. In addition, Kumar et al. evaluate and discuss our current understanding of the different functions of Vibrio pathogenicity island-1. In V. cholerae, this is related to virulence, important for toxin production, and essential for disease development. Biswas et al. summarize the diguanylate cyclases 
(DGCs) identified in $V$. cholerae so far, and emphasize the importance of DGCs and their product c-di-GMP in the virulence and lifecycle of $V$. cholerae.

Increased attention is being paid to the fact that the large number of microorganisms residing in human gastrointestinal tract establish a special micro-ecological system, which immediately responds to the invasion of $V$. cholera through the mechanism of "colonization resistance", such as the production of antimicrobial peptides, the competition of nutrients, and maintenance of the intestinal barrier. At the same time, $V$. cholerae can quickly sense these signals and regulate the expression of related genes to avoid stresses during infection, thereby successfully colonizing the surface of small intestinal epithelial cells. The comprehensive review by Qin et al. summarizes the interactions between the gut microbiota and $V$. cholerae in terms of type VI secretion system, quorum sensing, reactive oxygen species/pH stress, and bioactive metabolites.

\section{Vibrio parahaemolyticus VIRULENCE REGULATION AND HOST INTERACTIONS}

$V$. parahaemolyticus is a common pathogenic marine bacteria that can cause gastrointestinal infections and other health complications, posing a life threat to patients with weakened immune functions. In the past two decades, the pathogenicity of environmental $V$. parahaemolyticus has greatly increased. With more frequent studies showing the fast-evolving nature of $V$. parahaemolyticus, in-depth evaluation of its pathogenic ability becomes imperative. In this regard, Liu et al. demonstrate that the difference in pathogenicity of environmental $V$. parahaemolyticus strains is caused by a combination of HGT level, pathogenic elements distribution, and secretory system properties. Therefore, it is necessary to further study the genomic differences between environmental and clinical $V$. parahaemolyticus in order to better understand the pathogenicity of $V$. parahaemolyticus.

An in-depth understanding of the non-classical virulence genes of $V$. parahaemolyticus will help to better understand its pathogenic mechanism. Some manuscripts in this Research Topic reflect this view. For example, Pérez-Reytor et al. identify a gene encoding zonula occludens toxin (Zot) in the genome of highly cytotoxic strains of Chilean $V$. parahaemolyticus, which contributes to the virulence of $V$. parahaemolyticus through disturbance of the actin cytoskeleton. Yang et al. investigate the function of a prophage-related gene named VpaChn25_0724 in $V$. parahaemolyticus CHN25. Their results reveal that VpaChn25_0724 contributes to the cell membrane integrity and growth of $V$. parahaemolyticus.

\section{VIRULENCE REGULATION IN OTHER Vibrios}

$V$. harveyi, V. alginolyticus and V. vulnificus are the chief cause of vibriosis and have led to high mortality of marine animals and severe global economic losses. Understanding the virulence regulatory mechanisms of these Vibrios is the key to better development of fish vaccines. To this end, $\mathrm{Xu}$ et al. show that chemotactic genes che $A$, cheB, cheR, cheV and che $Y$ can regulate the adhesion ability of $V$. harveyi by affecting motility, and participate in the adjustment of adhesion at different temperatures, salinities and $\mathrm{pH}$. Zeng et al. analyze the succinylome of $V$. alginolyticus for the first time and reveal the possible biological effects of lysine succinylated protein, of which $7.5 \%$ is predicted to be a virulence factor so it may provide possible targets for the development of attenuated vaccines. Gong et al. reveal that V. vulnificus RpoS regulates TolCV1 expression, thereby affecting the secretion of RtxA1, bile salt tolerance and lethality in mice.

\section{VIRULENCE AND GENE DIVERSITY OF Vibrio spp}

Vibrios are an abundant and diverse group of bacteria in marine and estuarine environments, and there is evidence of biochemical and genotypic correlations with virulence potential. In this regard, Lydon et al. determine the biochemical characteristics and virulence genotypes of 30 clinical and 39 oyster isolates of $V$. vulnificus based on the types of $16 \mathrm{~S}$ rRNA gene $(\mathrm{rrn})$ and virulence-related gene $(v c g)$, which identify a relationship between isolates from the cooler season and systemic virulence potential in $V$. vulnificus. Besides, Wu et al. determine the genomic sequence of T3SS effector-encoding regions from 62 strains of four different species, and clarified the variation of their effector repertoires. In addition, they found a potential novel effector in $V$. harveyi and $V$. campbellii strain, and revealed that differences in T3SS-mediated cytotoxicity not only dependent on variation in the Vibrio T3SS effector repertoires, but also initial adhesion ability to host cells.

\section{CONCLUDING REMARKS}

The primary articles and reviews featured in this Research Topic are great examples presenting the current state of knowledge in this exciting and fast developing field of Vibrio spp. virulence regulation and host interactions. Through each new article, we gain a deeper understanding of the interesting and unique mechanisms controlling Vibrio pathogenicity and the pathogen-host interactions. In turn, these studies will contribute to the formulation of vibriosis control strategies and the development of highly effective live attenuated vaccines.

\section{AUTHOR CONTRIBUTIONS}

All authors listed have made a substantial, direct and intellectual contribution to the work, and approved it for publication.

\section{FUNDING}

This work was supported by grants from the Natural Science Foundation of Fujian Province under contract No. 2019J06020 
and the National Natural Science Foundation of China under contract No. 32173016.

Conflict of Interest: The authors declare that the research was conducted in the absence of any commercial or financial relationships that could be construed as a potential conflict of interest.

Publisher's Note: All claims expressed in this article are solely those of the authors and do not necessarily represent those of their affiliated organizations, or those of the publisher, the editors and the reviewers. Any product that may be evaluated in this article, or claim that may be made by its manufacturer, is not guaranteed or endorsed by the publisher.

Copyright $\odot 2021$ Huang, Fu, Sun and Brzostek. This is an open-access article distributed under the terms of the Creative Commons Attribution License (CC $B Y)$. The use, distribution or reproduction in other forums is permitted, provided the original author(s) and the copyright owner(s) are credited and that the original publication in this journal is cited, in accordance with accepted academic practice. No use, distribution or reproduction is permitted which does not comply with these terms. 